\title{
LOW-COST POSITIONING AND HEADING DETERMINATION SYSTEM: EXPERIMENTAL CLASSICAL 2D EKF SENSORS FUSION AND ACCURACY EVALUATION
}

Adrian Kaczmarek ${ }^{1}$, Witold Rohm ${ }^{1}$, Lasse Klingbeil², Janusz Tchórzewski ${ }^{3}$

${ }^{1}$ Institute of Geodesy and Geoinformatics, Wroclaw University of Environmental and Life Sciences, Wrocław, 50375, Poland

${ }^{2}$ Institute of Geodesy and Geoinformation, University of Bonn, Bonn, 53115, Germany

${ }^{3}$ Global Credit Development Fund Poland sp. z 0.0., Wrocław, 50072, Poland

Correspondence to: Adrian Kaczmarek¹ (adrian.kaczmarek@upwr.edu.pl)

https://doi.org/10.31490/9788024845050-7

\section{Abstract}

This research is focused on sensor fusion to determine the two-dimensional position and heading orientation using only low-cost sensors like GNSS receivers, IMU (without accelerometer and magnetometer), and odometry. In this task, we used classical extended Kalman Filter with additional observations as a control input (angular rate and odometry distance). The combination of high stability GNSS observation over time with short-term high accuracy IMU and odometry gives us a very accurate position and heading after fusion. As a result, we obtained a 0.62-degree accuracy for heading, $3.9 \mathrm{~cm}$, and $2.2 \mathrm{~cm}$ for the position (North and East respectively). Additionally, we noticed and resolved a problem with time synchronization between sensors which is very important to get high-standard results (accuracy better than one decimetre).

Keywords: EKF, low-cost sensors, IMU, heading, integration, u-blox, xsens, GNSS, odometry

\section{INTRODUCTION}

Nowadays we have a lot of different manufacturers of low-cost MEMS (Micro Electro Mechanical System) sensors that are used in smartphones, watches, survey equipment (pole tilt compensation of GNSS receivers), robotics, etc. The low-cost sensors have different accuracy, measurement range, noise level, stability, and quantity of degrees of freedom (DOF) in one chip-structure for example 3, 6, and 9 DOF. The most popular sensors in their structures have a 3-axis gyroscope, 3-axis accelerometer, and 3-axis magnetometer. Apart from inertial MEMS sensors there are low-cost GNSS receivers produced by u-blox (M8P (L1) and F9P (L1/L2)), which provide high accurate horizontal position in RTK Fix mode (1-2.5 cm - (u-blox notes)). The major challenge in low-cost sensor fusion is that, these sensors have poor stability, big drift, and noise in time (when used without updates e.g. from GNSS (Global Navigation Satellite System), odometry, etc.), and for the most part, are not calibrated by the producer. One of the integration methods is the AHRS (Attitude Heading Reference System) which joins the 9 DOF Madwigck et al. (2010) and Mahony et al. (2008) approaches. These methodologies give us about $1-3^{\circ}$ heading 
accuracy when the sensor is in a static position (Li et al. 2006; Sheng and Zhang 2013). Horizontal position accuracy after sensor fusion is mainly dependent from GNSS position error, quality IMU data (Inertial Measurement Unit), and used methodologies. In the paper Rios and White (2002), the authors indicate that position after sensor fusion is about $1 \mathrm{~m}$ accuracy (when the temporarily lack of GNSS signal is over $60 \mathrm{~s}$ ) and heading accuracy is better than $0.5^{\circ}$ in the dynamic state. It should be noted that the authors used a commercial inertial system.

Another potentially useful sensor to stabilize the orientation solution is magnetometer. However, magnetometer is a very sensitive to interference with external electromagnetical fields. Potential source of interefernce are: electric engine (the field strength depends on the load on the electric motor e.g. in lawnmower), metal elements (mesh fence, power supply) and many others derived from ferromagnetic materials and electronic devices. 'The magnetometer could be calibrated, but if we have a changeable electromagnetic field in time and we do not know the distribution of the electromagnetic field,such excercise won't be successful for high-quality applications. .

Our research is focused on the integration of low-cost two GNSS receivers, IMU (1 DOF) and odometry (optical encoder) to get a high accurate horizontal position and long-term orientation stability (without magnetometer) for an autonomous lawnmower. For the integration, we used synchronous loosely coupled extended Kalman Filter (EKF) which combing heading and position in one calculating process.

\section{INPUT DATA}

The data from low-cost sensors have been collected using our test platform (Fig. 1). This platform apart from low-cost system includes professional mobile mapping equipment: Applanix POS LV 120 V5 which provides a high accurate heading and position. Our test platform also includes: microcomputer Raspberry Pi 3, two GNSS RTK receivers (u-blox F9P), IMU (xsens MTi-7), odometer (optical encoders) mounted on two wheels, and geodetic precise $360^{\circ}$ prism to measure reference position by the robotic total station (Trimble S8). The rate and unit of the data type are showed in Table 1. Data from Applanix and total station was used only as a reference for heading and position.

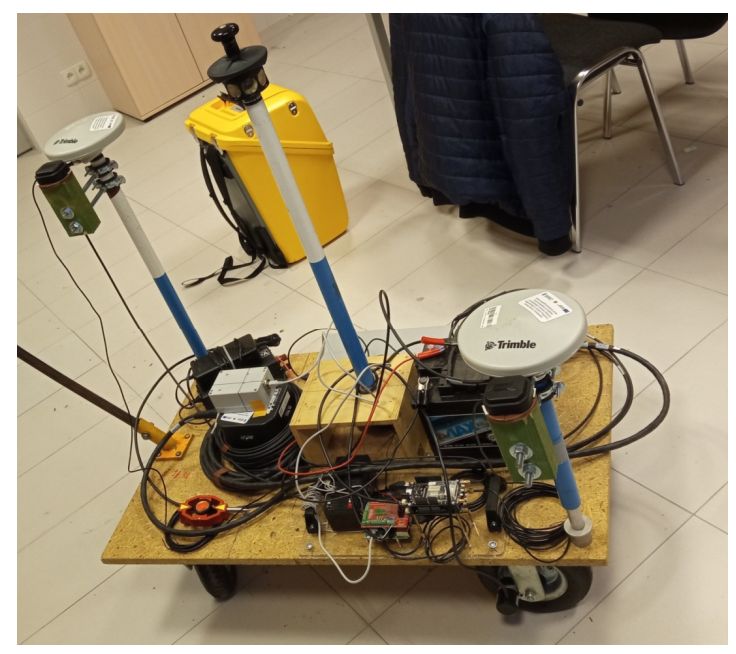

Fig. 1. Test platform 
Table 1. Data collected by the test platform

\begin{tabular}{llll}
\hline Sensor & Data type & Unit & Rate \\
\hline u-blox F9P & horizontal position & {$[\mathrm{m}]$} & $5 \mathrm{~Hz}$ \\
\hline xsens & z-axis angular rate & {$[\mathrm{rad} / \mathrm{s}]$} & $50 \mathrm{~Hz}$ \\
\hline Odometer & distance & {$[\mathrm{m}]$} & $50 \mathrm{~Hz}$ \\
\hline $\begin{array}{l}\text { Applanix } \\
\text { (only reference data) })\end{array}$ & horizontal position & {$[\mathrm{m}]$} & $200 \mathrm{~Hz}$ \\
\hline $\begin{array}{l}\text { Total station } \\
\text { (only reference data) }\end{array}$ & horizontal position & {$[\mathrm{m}]$} & $200 \mathrm{~Hz}$ \\
\hline
\end{tabular}

The data from Applanix has been calculated in post-processing mode in POSPac MMS software which is dedicated to the Applanix system. Fig. 2 shows a test area. It is a terrace one of the Wroclaw University of Environmental and Life Sciences (UPWr) building (Wrocław, Poland).

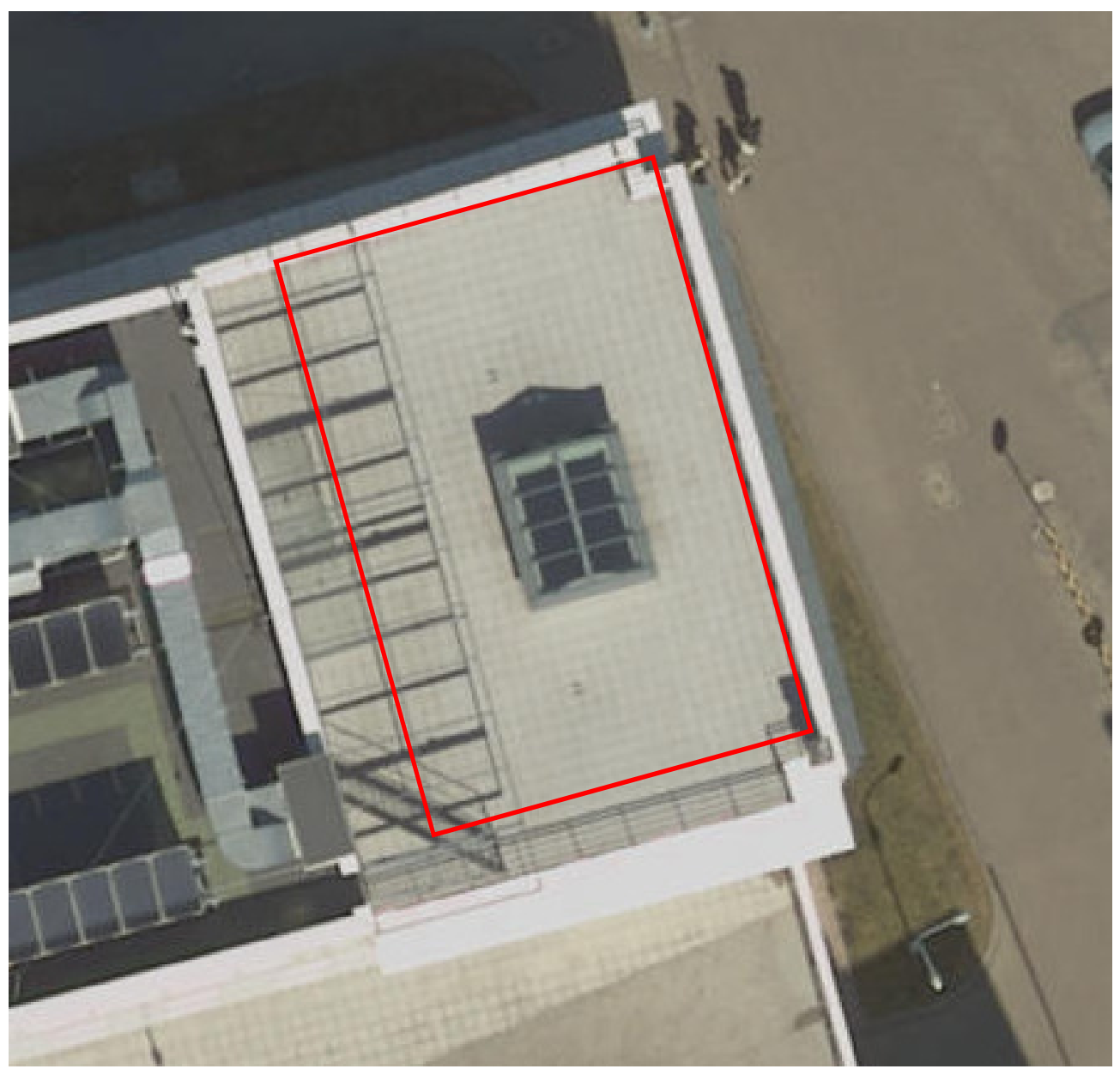

Fig. 2. Test area - terrace of one of the building UPWr (Wrocław, Poland)

\section{METHODOLOGY}

The strategy is focused on the known loosely coupled extended Kalman Filter (nonlinear filter) but in this filter, we used gyroscope angular rate and odometry distance as a control input (in prediction step eq. (1) and (2)), we also added a system noise to these sensors. Update steps include Kalman gain (3), position and heading update (4), and covariance matrix update (5). 
The IMU sensor has high accuracy but is not stable in in time. Contrary, azimuth from two GNSS antennas has poor accuracy but relatively long-term stability. If we join these two sensors we get high accuracy and orientation stability. Authors sensors fusion algorithm are shown in equations (1) - (10) based on Greval et al. 2020.

Prediction step (equation $1-5$ ):

$$
\begin{aligned}
& x_{k}^{-}=f\left(x_{k-1}, u_{k}, w_{k}\right)=\left[\begin{array}{l}
x_{k} \\
y_{k} \\
\varphi_{k}
\end{array}\right]=\left[\begin{array}{c}
x_{k-1}+\cos \left(\varphi_{k-1}\right) \cdot\left(\Delta o d o_{k}+w_{\text {odo }}\right) \\
y_{k-1}+\sin \left(\varphi_{k-1}\right) \cdot\left(\Delta o d o_{k}+w_{\text {odo }}\right) \\
\varphi_{k-1}+\left(\omega_{\text {gyro }, k}+w_{\text {gyro }}\right) \cdot \Delta t
\end{array}\right] \\
& P_{k}^{-}=\Phi P_{k-1} \Phi^{T}+G Q G^{T}
\end{aligned}
$$

Transition matrix:

$$
\Phi_{k-1}=\frac{\partial f(x, u, w)}{\partial x}=\left[\begin{array}{ccc}
1 & 0 & -\sin \left(\varphi_{k-1}\right) \cdot \Delta o d o_{k} \\
0 & 1 & \cos \left(\varphi_{k-1}\right) \cdot \Delta o d o_{k} \\
0 & 0 & 1
\end{array}\right]_{x=x_{k-1}, u=u_{k-1}}
$$

System noise:

$$
\begin{aligned}
& Q=\left[\begin{array}{cc}
\sigma_{\text {odo }}^{2} & 0 \\
0 & \sigma_{\text {gyro }}^{2}
\end{array}\right] \\
& G=\frac{\partial f(x, u, w)}{\partial w}=\left[\begin{array}{cc}
\cos \left(\varphi_{k-1}\right) & 0 \\
\sin \left(\varphi_{k-1}\right) & 0 \\
0 & \Delta t
\end{array}\right]_{x=x_{k-1}, u=u_{k-1}}
\end{aligned}
$$

Update step (equation $6-10$ ):

$$
\begin{aligned}
& K_{k}=P_{k}^{-} H^{T}\left(H P_{k}^{-} H^{T}+R\right)^{-1} \\
& x_{k}=x_{k}^{-}+K_{k}\left(z_{k}-h\left(x_{k}^{-}\right)\right) \\
& P_{k}=\left(I-K_{k} H\right) P_{k}^{-}
\end{aligned}
$$

Measurement Vector:

$$
z_{k}=\left[\begin{array}{l}
x_{G P S, k} \\
y_{G P S, k} \\
\varphi_{G P S, k}
\end{array}\right]
$$

Measurement Noise:

$$
R=\left[\begin{array}{ccc}
\sigma_{G P S, X}^{2} & 0 & 0 \\
0 & \sigma_{G P S, Y}^{2} & 0 \\
0 & 0 & \sigma_{G P S, \varphi}^{2}
\end{array}\right]
$$

where:

$x_{k}^{-}$- prediction state vector (X,Y, heading), $u_{k}$ - control input values (angular rate $(\omega)$, odometry distance (odo)), $w_{k}$ - noise of control input, $P_{k}^{-}$- prediction covariance matrix, $K_{k}$ Kalman gain, $x_{k}$ - update state vector, $P_{k}$ - update covariance matrix, $R$ - measurement noise 
(position and heading), $\Phi$ - transition matrix, $Q$ - control input noise matrix, $G$ - system noise of control inputs, $z_{k}$ - measurement values (position and azimuth from two GNSS antennas).

Noise values for all sensors have been assigned from device datasheets.

\section{RESULTS}

In the first step we determined the accuracy of the Applanix system referenced to the independent total station measurement. In Table 2 we can see that accuracy of the Applanix mobile mapping system is sufficient to act as a reference in further studies. As a consequence, data from Applanix was used for further analysis because this reference track is denser $(200 \mathrm{~Hz})$ than tachymetry $(1 \mathrm{~Hz})$.

Table 2. Position and heading accuracy of Applanix mobile mapping system

\begin{tabular}{ll}
\hline Direction & Applanix position accuracy [RMS] \\
\hline North & $0.014 \mathrm{~m}$ \\
\hline East & $0.013 \mathrm{~m}$ \\
\hline Heading & 0.1 degree (value after post-processing in POSPac software) \\
\hline
\end{tabular}

Fig. 3 shows results of sensor fusion for heading (GNSS azimuth and IMU). In this figure, we can see high accuracy heading stability. Additionally, Fig. 4 shows azimuth residuals before and after sensors fusion. Azimuth accuracy is shown in Table 3. In the Table 3 we also can see that heading accuracy is $5.55 \mathrm{deg}$. This is due to the fact that in different terrain conditions we have Float (crossing the walls, garden with tall trees, shrubs etc.) or Fix (open sky) type RTK solution. Changing of the type RTK solution causes deterioration of the position accuracy (up to $20-50 \mathrm{~cm}$ ) on at least one of the antennas and next deterioration of the heading accuracy. Distance between two GNSS antennas is $1.13 \mathrm{~m}$. The tests were done there where was not huge impact of the electromagnetic field on GNSS signal like high voltage lines. The main cause worse heading accuracy was type of the RTK solution.

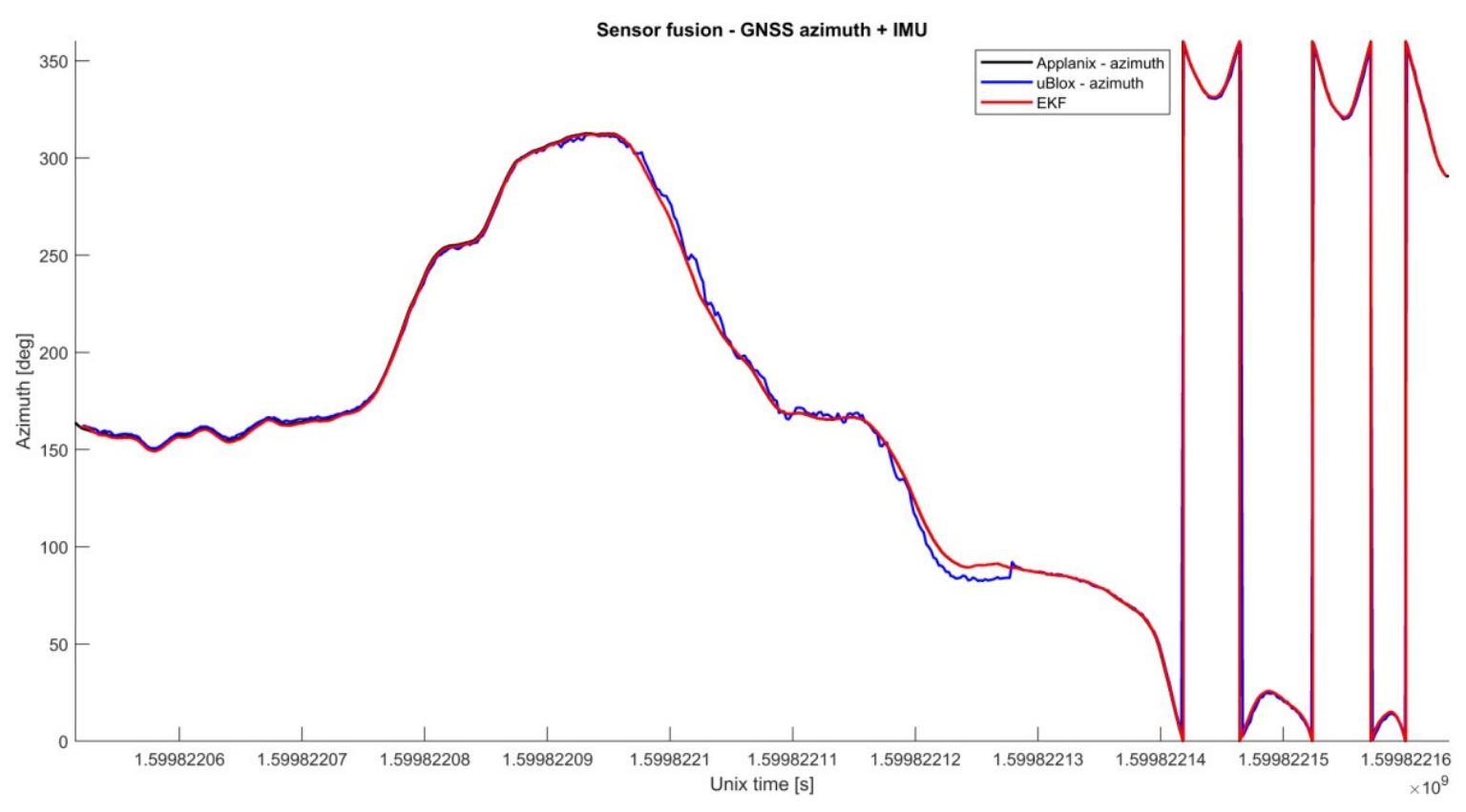

Fig. 3. GNSS heading and IMU sensors fusion results 

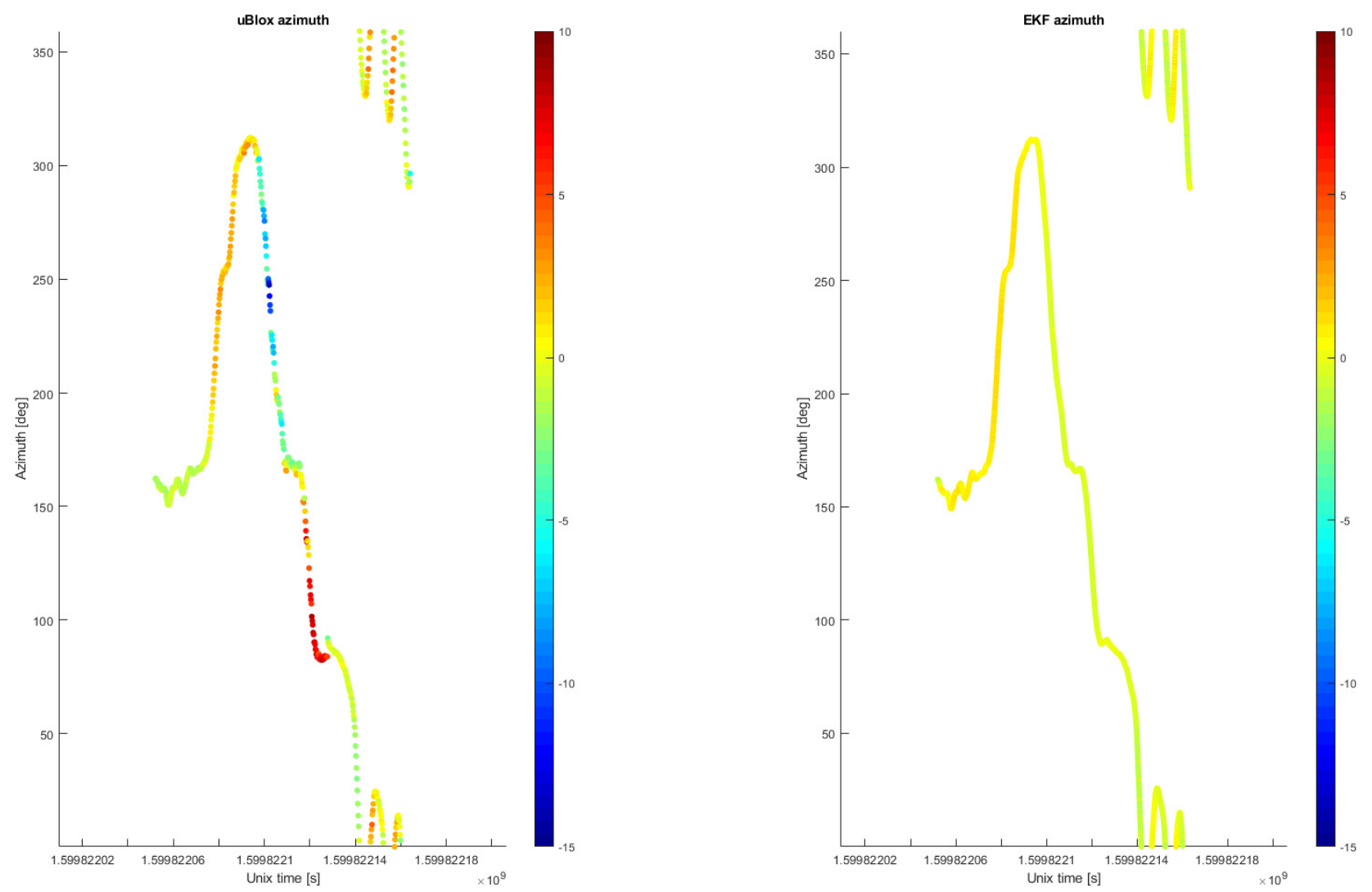

Fig. 4 Heading residuals before (left) and after (right) sensors fusion (colorbars unit: degrees).

Table 3. Accuracy of heading integration (reference heading = Applanix)

\begin{tabular}{ll}
\hline Heading from: & Heading accuracy [RMS] \\
\hline $\begin{array}{l}\text { two GNSS antennas } \\
\text { (u-blox) }\end{array}$ & 5.55 degrees \\
\hline sensors fusion (EKF) & 0.62 degrees \\
\hline
\end{tabular}

Table 4 and Fig. 5 shows position accuracy after sensor fusion. In Fig. 5 we also can see that (black arrow) our system had the problem with time synchronization between sensors and is therefore showing deviation between EKF solution and Reference. While in Fig. 6 we can see position error before and after sensors fusion. Additionally, on Fig. 6 (left side) we see places where was RTK Float solution (deterioration of uBlox position accuracy). 


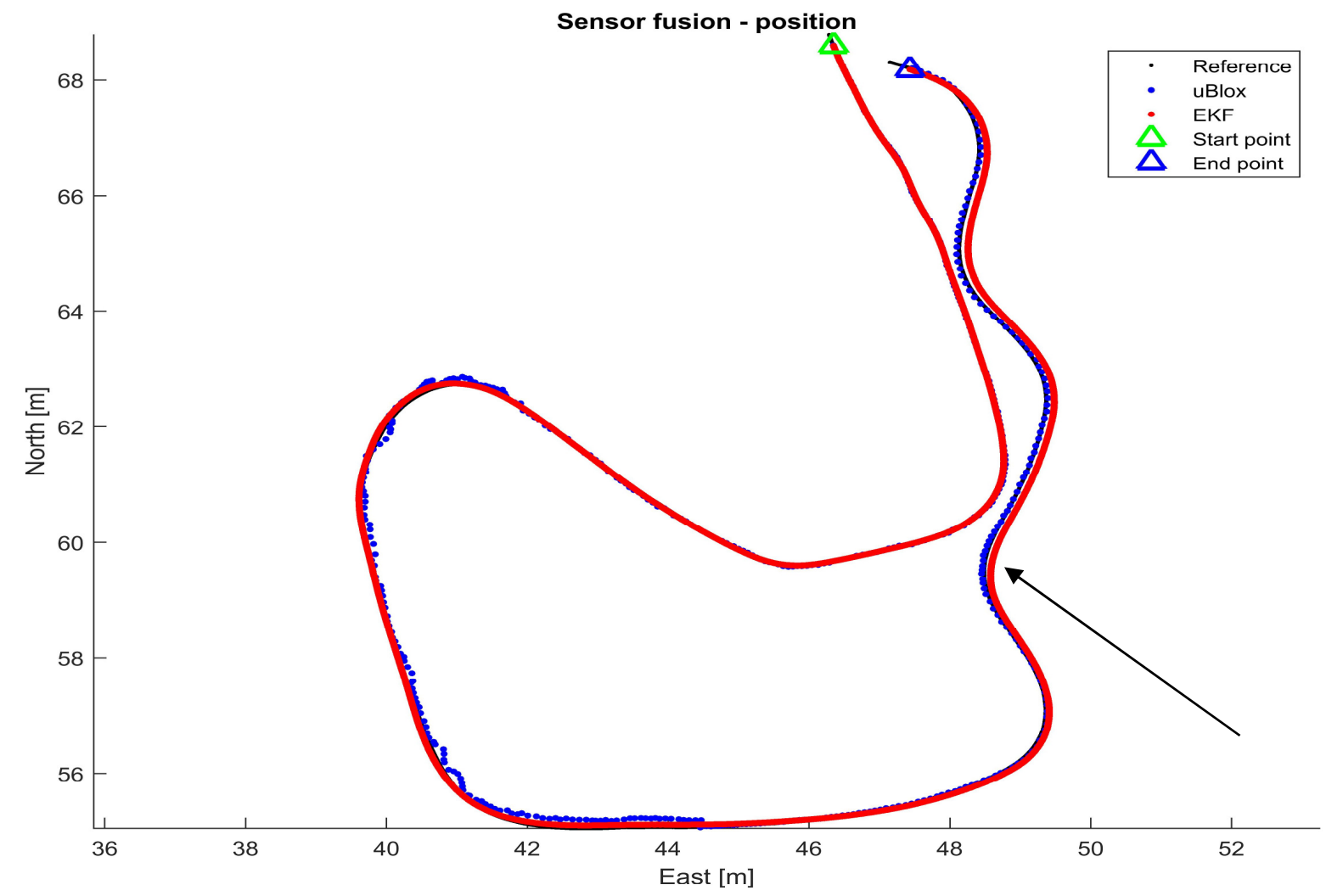

Fig. 5. Position after sensor fusion (GNSS+IMU+odometry). The black arrow shows probably starts a problem with time synchronization between sensors (drift cumulative of Raspberry Pi Real-Time Clock (RTC)).
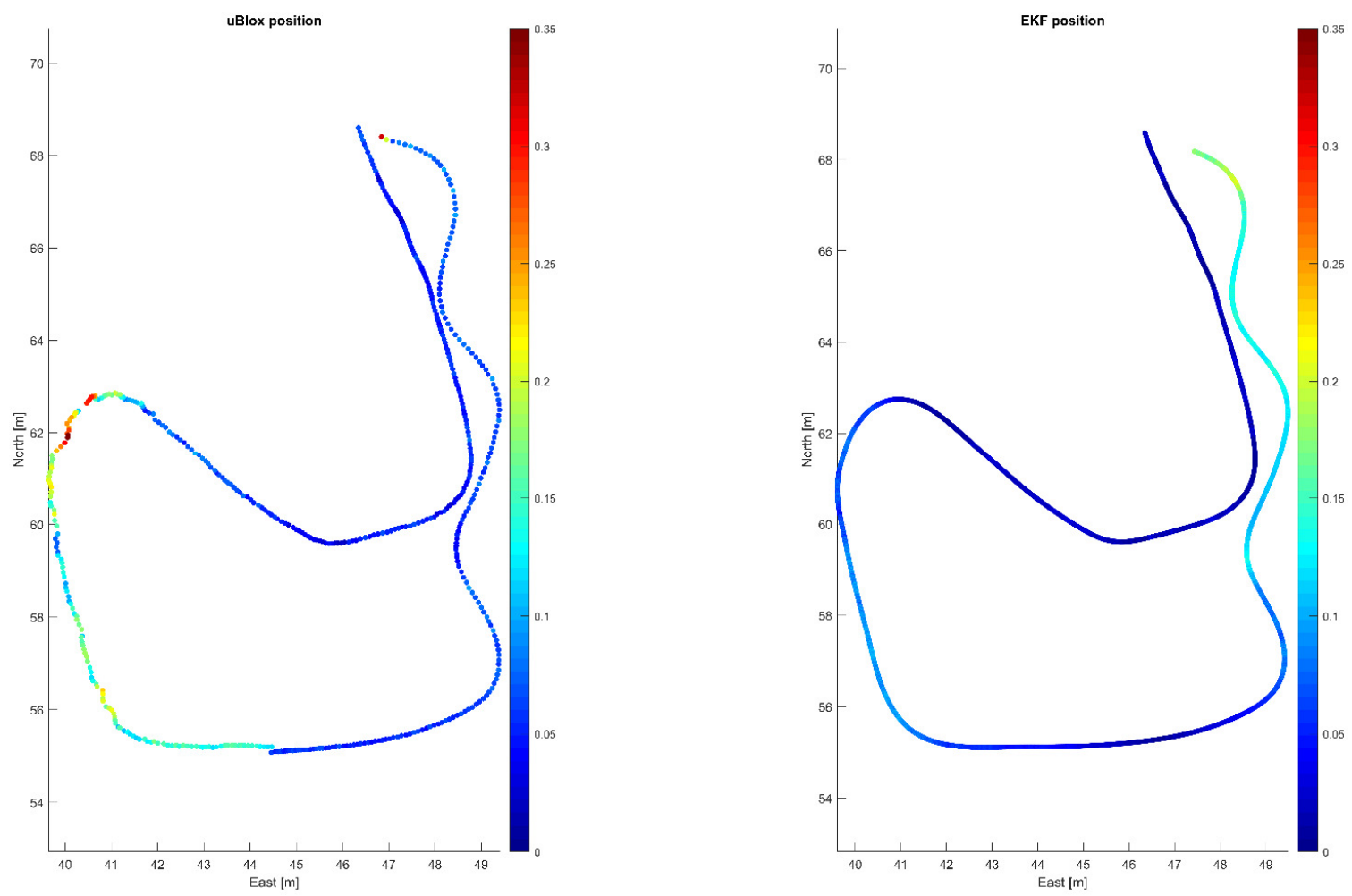

Fig. 6 Position error before (left) and after (right) sensors fusion (colorbars units: meters [m]) 
Table 4. Accuracy of position integration (reference position = Applanix)

\begin{tabular}{lll}
\hline Position from: & Direction & Position accuracy [RMS] \\
\hline u-blox & North & $0.052 \mathrm{~m}$ \\
& East & $0.089 \mathrm{~m}$ \\
\hline \multirow{2}{*}{ sensors fusion (EKF) } & North & $0.039 \mathrm{~m}$ \\
& East & $0.022 \mathrm{~m}$ \\
\hline
\end{tabular}

\section{CONCLUSIONS AND FURTHER WORK}

When analyzing the obtained sensors fusion results, we find that the low-cost sensor like ublox RTK, xsens IMU, and odometry can be used to precise positioning and orientation determination. After integration, we obtained almost two times better results for position and orientation than without integration (Table 3 and 4). It should be noted that the precise time synchronization between the sensors is very important. Built-in cheap real-time clocks (like in Raspberry Pi, PC, etc) almost always have a large drift. Further work will be focused on improving hardware: synchronization sensors using a signal from 1PPS pin from ublox module. This pin we can configure to work with a different frequency which can trigger all sensors at this same time. Another option is to add OCXO (Oven Controlled Crystal Oscillator) or TCXO (Temperature Compensated Crystal Oscillator) generator to improve measuring time by Raspberry $\mathrm{Pi}$.

Funding: The research is co-financed under the Leading Reasarch Groups support project from the subsidy increased for the period 2020-2025 in the amount of $2 \%$ of the subsidy referred to Art. 387 (3) of the Law of 20 July 2018 on Higher Education and Science, obtained in 2019, and a project "Testing and optimizing the platform performance for precise positioning" grant no. Z030/0025/20 (B090/0052/19).

\section{REFERENCES}

Grewal, M. S., Andrews, A. P., and Bartone, C. G. (2020). Global navigation satellite systems, inertial navigation, and integration. John Wiley \& Sons.

R. Mahony, T. Hamel, and J.-M. Pflimlin. (2008) "Nonlinear Complementary Filters on the Special Orthogonal Group," IEEE Transactions on Automatic Control, Institute of Electrical and Electronics Engineers (IEEE), pp. 1203-1217.

Rios, Jose A. and White, Elecia. (2002) "Fusion Filter Algorithm Enhancements For a MEMS GPS/IMU," Proceedings of the 2002 National Technical Meeting of The Institute of Navigation, San Diego, CA, January 2002, pp. 126-137.

Sheng, H. L., and Shang, T. H. (2013). "Low-cost AHRS design based on extending Kalman filter", LIU Dong-dong; College of Energy and Power, Nanjing University of Aeronautics and Astronautics, 10.

S. Madgwick (2010), "An Efficient Orientation Filter for Inertial and Inertial/Magnetic Sensor Arrays," Report x-io and University of Bristol

Y. Li, A. Dempster, B. Li, J. Wang and C. Rizos. (2006) "A low-cost attitude heading reference system by combination of GPS and magnetometers and MEMS inertial 
sensors for mobile applications," Positioning, Vol. 1 No. 10

\section{TECHNICAL NOTES}

u-blox. https://www.u-blox.com/sites/default/files/NEO-M8P_DataSheet_\%28UBX$15016656 \% 29 . p d f$

u-blox. https://www.u-blox.com/sites/default/files/ZED-F9P_DataSheet_\%28UBX17051259\%29.pdf 